\title{
MARKET DEMAND IS A CONTINUOUS FUNCTION OF PRICES
}

\author{
Walter TROCKEL \\ Lniversing of Bonn, 5300 Bonn, West Germany
}

Received 23 November 1982

A natural class of probabilities on the space of consumers' preferences is presented for which market (i.e.. mean) demand is a (continuous) function of prices although individual preferences may be non-convex.

\section{Introduction}

There is a qualitative difference between individual demand and market demand. This is thoroughly discussed in Mantel (1974) and Shafer and Sonnenschein (1982). While certain structures of individual demand are lost in the process of aggregation others may be gained [cf. Hildenbrand (1982)]. The least that should be expected from aggregation is a continuous market demand function even if individual agents may have non-convex preferences and, therefore, set-valued demands. The importance of uniqueness and, therefore - since demand correspondences are upper hemi-continuous - continuity of market demand has been sufficiently motivated elsewhere [cf. Allen (1980), Araujo and Mas-Colell (1978). Dierker et al. (1980a,b), Hildenbrand (1980), Sondermann (1975)].

In the present paper we shall present a natural class of probabilities on the space of preference relations yielding continuous mean demand functions which is dense in the set of all probabilities.

The characteristic function of our approach relies on a natural parametrization of preferences by prices which allows via Fubini's Theorem the consideration of probabilities whose support is the whole space of preferences. The idea of 'suitable diffusion' as a necessary condition for 
uniqueness of market demand as expressed in Debreu (1972) and Hildenbrand (1974) is given a concrete formalization. The 'uniform' or 'equal distribution' which cannot be modelled on the huge space of preferences is weakened to 'equal distribution' of any kind of demand behavior over the space of budgets. Our concept of budget dispersion. which was introduced by Dierker et al. (1981). defines a class of probabilities with the property that similar demand behavior occurs in similar budget situations with similar probabilities.

\section{The model}

We consider $l \geq 2$ perfectly divisible commodities. The consumption set for every consumer is $\mathbf{R}_{+}^{l}$, the non-negative orthant of the commodity space $\mathbf{R}^{\prime}$. Paying regard to the homogeneity of demand we normalize budgets by assuming all consumers' wealth, $w$, to be equal to one. Accordingly, we choose as price space $P=\left\{p \in \mathbf{R}^{\prime} \mid p \gg 0\right\}$. Let $\%$ denote the set of complete, weakly monotone, continuous preorderings on $\mathbf{R}_{+}^{\prime}$. called preference relations, $\succsim \in \mathbb{P}$. The set $\%$ endowed with the topology of closed convergence [cf. Hildenbrand (1974, p. 96)] is a Polish space. i.e., a topologically complete, separable, metrizable space.

The demand set of an agent described by his wealth, $w=1$ and a preference relation $\succsim$ at a price system $p$ is

$\varphi(\succeq p)=\left\{x \in \mathbf{R}_{+}^{\prime} \mid p x \leq 1,(y \geq x) \Rightarrow p y>1\right\}$,

the set of $\succsim$-maximal elements in the budget set $\left\{x \in \mathbf{R}_{+} \mid p x \leq 1\right\}$. The demand correspondence

$\varphi: \varphi \times P \rightarrow \mathbf{R}_{+}:(\succsim, p) \mapsto \varphi(\succsim, p)$

is upper hemicontinuous (u.h.c.). For any $p \in P$ this defines an u.h.c. correspondence $\varphi(\cdot, p): \varphi \rightarrow \mathbf{R}_{+}^{\prime}$ which has a measurable graph [cf. Hildenbrand (1974, p. 102)].

To define mean (i.e., market) demand we need a probability measure $\mu$ on the $\sigma$-field $9(9)$ of Borel subsets of $\%$. The market demand at the price system $p$ is

$$
f_{\mu}(p)=\int_{Q p} \varphi(\succeq, p) \mathrm{d} \mu
$$


For the concept of the integral of a correspondence see Hildenbrand (1974, p. 53). The price space $P$ has in a natural way the structure of a $\sigma$-compact, locally compact commutative group. The group operation is coordinatewise multiplication. Formally, for $q, q^{\prime} \in P$ define $q \cdot q^{\prime}=$ $\left(q_{1} q_{1}^{\prime}, \ldots, q_{l} q_{l}^{\prime}\right)$. Also by coordinatewise multiplication we get an action of the group $P$ on the consumption set $\mathbf{R}_{+}^{\prime}$.

$P \times \mathbf{R}_{+} \rightarrow \mathbf{R}_{+}:(q, x) \mapsto x^{q}=\left(q_{1} x_{1}, \ldots, q_{l} x_{l}\right)$.

Now define for any $z \in \mathscr{P}$ and $q \in P$ the preference relation $\succsim_{q} \in \mathscr{P}$ by $x^{q} \gtrsim_{q}{ }^{q}: \Leftrightarrow x \succsim y$.

Now consider the action $a$ of $P$ on $P$ defined by

$\boldsymbol{a}: P \times \mathscr{P} \rightarrow \mathscr{P}:(q, \succsim) \rightarrow \succsim_{q}$.

The crucial consequence for demand is represented by the following formula:

$q \cdot \varphi(\succeq p)=\varphi\left(\succsim_{q}, q^{-1} \cdot p\right), \quad$ or, equivalently

$q \cdot \varphi(\succeq q \cdot p)=\varphi\left(\succsim_{q}, p\right)$.

This suggests that integrating demand at $p$ over all preferences $\succsim_{q}$, $q \in P$. can be done by integrating demands at $q \cdot p$ for a fixed preference over prices $q \in P$. Indeed, this can be done, and we shall show now how it works.

Following Furstenberg (1971) we define now a generalization of the convolution of measures for the case, that the two involved measures are not defined on the same space.

Let $\xi$ and $\mu$ be measures on $\mathscr{B}(P)$ and $\mathscr{B}(\mathscr{P})$, respectively. Let $\xi \otimes \mu$ be the product measure on $\mathscr{h}(P \times \mathscr{P})$. The convolution $\xi * \mu$ is defined as the image measure of $\xi \otimes \mu$ under the mapping $a$, i.e., $\xi * \mu=\xi \otimes \mu \circ a^{-1}$.

We can state now our dispersion concept which is a slight modification of price-dispersion introduced in Dierker et al. (1980a, b). It relies on the fact that on the group $P$ of prices there exists an invariant measure which is (up to normalization) also unique. Such a measure is called a Haar measure. 
Definition. A probability $\mu^{*}$ on the space 9 of preferences is called $P$-dispersed, if there is a probability on $(P$, $(P))$ which is absolutely continuous with respect to the Haar measure on $(P, 6(P))$ and some

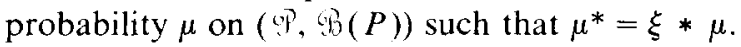

Note that the set of $P$-dispersed probabilities on $y$ is a dense subset of the space of probabilities on with the narrow (or weak) topology.

\section{Result}

Theorem. Let $\mu^{*}$ be a P-dispersed probability on (9. 9 . (9)). Then the market demand relation $f_{\mu^{*}}: P \rightarrow \mathbf{R}_{+}^{\prime}$ defined by $f_{\mu^{*}}(p)=\int_{w *} \varphi(\succsim, p) \mu^{*}(\mathrm{~d} \succeq)$ is a continuous function.

Proof. By definition of $\mu^{*}$ we get

$$
\int_{Q \varphi} \varphi(\succeq, p) \mu^{*}(\mathrm{~d} \succsim)=\iint_{\varphi \varphi} \varphi\left(a\left(\succsim^{\prime}, q\right), p\right) \xi(\mathrm{d} q) \mu\left(\mathrm{d} \succsim^{\prime}\right) .
$$

It suffices to show that for any $p \in P$ the inner integral is a singleton. A necessary and sufficient condition for this is that for any $p \in P$

$\xi\left(\left\{q \in P \mid \# \varphi\left(a\left(z^{\prime}, q\right), p\right)>1\right\}\right)=0$.

Here \# denotes cardinality. Now, since $\varphi\left(a\left(\succeq^{\prime}, q\right), p\right)=\varphi\left(\succeq_{q}^{\prime}, p\right)=$ $q \circ \varphi\left(\succsim^{\prime}, p\right)$, we get $\# \varphi\left(a\left(z^{\prime}, q\right), p\right)=\# \varphi\left(z^{\prime}, q \cdot p\right)$. Hence we are finished if for $\mu$-almost all preferences $\succsim \in \in$ we can show that

$\xi(\{q \in P \mid \# q(\succsim, q \circ p)>1\})=0$.

But, since $\xi$ is absolutely continuous with respect to the Haar measure on $P$ and therefore also with respect to the Lebesque measure on $P$. this can be concluded directly from Lemma 1, p. 593 in Mas-Colell and Neuefeind (1977). Q.E.D.

For a related result in the general context of mean maximizers see Trockel (1983). 


\section{Concluding remarks}

Clearly, the same result can be proved if wealth varies and prices are normalized in some way. The acting group of prices respectively budgets describes those aspects of preferences, diffusion or 'equal distribution' which are needed. The 'equal distribution' is formalized by the Haar measure on the acting group. This approach provides a general and natural method to formalize dispersion of certain aspects on large measure spaces on which there is not enough structure to define a notion of an invariant measure.

The present dispersion concept has been used in Dierker et al. (1981) to define classes of measures on consumers' characteristics yielding continuously differentiable market demand functions. It is very likely that the present dispersion concept can also be used to bring about structural restrictions for market demand functions. Also formalization of diversification of information might possibly be approached in a similar way.

It might be interesting for the reader to compare the present result and the dispersion assumption used to derive it, with the reasoning used by Cournot (1838) to justify the assumption of a continuous market demand function as opposed to non-continuous individual demand [Cournot (1838, ch. IV, sect. 22), Walras $(1874,1877)]$ referring to this reasoning of Cournot ascribed the continuity of market demand to the 'law of large numbers'. Indeed, one may consider Birkhoff's pointwise ergodic theorem as a very general version of the law of large numbers. But it is just the ergodicity of the actions of group $P$ restricted to the orbits $P \circ \gtrsim$, which allows the representation of mean demand by the double integral in the second line of our proof. The classical 'space average equals time average' of statistical mechanics reappears in our context as 'space average equals price average' where 'space' means a space of preferences. The possibility of a natural decomposition of 9 in $P$-ergodic components is the reason for the uniqueness of market demand [cf. Trockel (1983)].

\section{References}

Allen, B., 1981, ^ nonparametric approach to smoothing by aggregation over preferences, Journal of Mathematical Economics, forthcoming.

Araujo, A. and A. Mas-Colell, 1978, Notes on the smoothing of aggregate demand, Journal of Mathematical Economics 5, 113-122.

Cournot, A., 1838, Recherches sur les principes mathématiques de la théorie des richesses 
Paris). (Librairie des sciences politiques et sociales, M. Rivière. Translated by Nathaniel T. Bacon, 1897, Researches into the mathematical principles of the theory of wealth (MacMillan, New York).

Debreu, G., 1972, Smooth preferences, Econometrica 40, 603-615.

Dierker, E., H. Dierker and W. Trockel, 1980a, Continuous mean demand functions derived from nonconvex preferences, Journal of Mathematical Economics 7. 27-33.

Dierker, E., H. Dierker and W. Trockel, 1980b, Smoothing demand by aggregation with respect to wealth, Journal of Mathematical Economics 7, 227-247.

Dierker, E., H. Dierker and W. Trockel, 1981, Price-dispersed preferences and $\mathrm{C}^{\prime}$ mean demand, SFB 21 Projektgruppe 'Theoretische Modelle', Discussion paper no. 88. University of Bonn.

Furstenberg, H., 1971, Random walks and discrete subgroups of Lie groups, in: Peter Ney. ed., Advances in probability (Marcel Dekker, New York).

Hildenbrand, W., 1974, Core and equilibria of a large economy (Princeton University Press, Princeton, NJ).

Hildenbrand, W., 1980, On the uniqueness of mean demand for dispersed families of preferences, Econometrica 48, 1703-1710.

Hildenbrand, W., 1982. Law of demand, SFB 21 Projektgruppe 'Theoretische Modelle', Discussion paper no. 77, University of Bonn, to appear in Econometrica.

Mantel, R., 1974, On the characterization of aggregate excess demand, Journal of Economic Theory 7, 348-353.

Mas-Colell, A. and W. Neuefeind, 1977, Some generic properties of aggregate excess demand and an application, Econometrica 45, 591-599.

Shafer, $W$. and $H$. Sonnenschein, 1982, Market demand and excess demand function, in: $K$. Arrow and M. Intriligator, eds., Handbook of mathematical economics (North-Holland, Amsterdam).

Sondermann, D., 1975, Smoothing demand by aggregation, Journal of Mathematical Economics 2, 201-224.

Trockel, W., 1983, Uniqueness of mean maximizers via an ergodic theorem. Mathematische Operationsforschung und Statistik, Series Optimization, forthcoming.

Walras, L., 1874, 1877, Elèments d’économie politique pure Lausanne, L. Corbaz, English translation by W. Jaffé, 1954, Elements of pure economies (Allen and Unwin, London). 